\title{
Thermodynamics/Dynamics Coupling in Weakly Compressible Turbulent Stratified Fluids
}

\author{
Rémi Tailleux \\ Department of Meteorology, University of Reading, Earley Gate, P.O. Box 243, Reading RG6 6BB, UK \\ Correspondence should be addressed to Rémi Tailleux, r.g.j.tailleux@reading.ac.uk
}

Received 6 December 2011; Accepted 22 January 2012

Academic Editors: E. Curotto and N. Marmier

Copyright () 2012 Rémi Tailleux. This is an open access article distributed under the Creative Commons Attribution License, which permits unrestricted use, distribution, and reproduction in any medium, provided the original work is properly cited.

In traditional and geophysical fluid dynamics, it is common to describe stratified turbulent fluid flows with low Mach number and small relative density variations by means of the incompressible Boussinesq approximation. Although such an approximation is often interpreted as decoupling the thermodynamics from the dynamics, this paper reviews recent results and derive new ones that show that the reality is actually more subtle and complex when diabatic effects and a nonlinear equation of state are retained. Such an analysis reveals indeed: (1) that the compressible work of expansion/contraction remains of comparable importance as the mechanical energy conversions in contrast to what is usually assumed; (2) in a Boussinesq fluid, compressible effects occur in the guise of changes in gravitational potential energy due to density changes. This makes it possible to construct a fully consistent description of the thermodynamics of incompressible fluids for an arbitrary nonlinear equation of state; (3) rigorous methods based on using the available potential energy and potential enthalpy budgets can be used to quantify the work of expansion/contraction $B$ in steady and transient flows, which reveals that $B$ is predominantly controlled by molecular diffusive effects, and act as a significant sink of kinetic energy.

\section{Introduction}

A large class of fluid flows of interest in traditional and geophysical fluid dynamics are characterised by fluid velocities much smaller than the speed of sound (low Mach number), strong density stratification yet with small relative density variations, and high Reynolds number (i.e., they are turbulent). It has become common practice to regard such flows as incompressible or nearly incompressible, and to describe them by means of particular approximations to the fully compressible Navier-Stokes equations known as the Boussinesq and anelastic approximations. Traditionally, such approximations are derived in the idealised context of purely adiabatic motions, hence excluding irreversible processes such as molecular viscous and diffusive processes. The equation of state is also usually linearised, so that density anomalies become proportional to temperature anomalies. In that case, it is easily shown that the resulting approximations filter out acoustic waves and thus fully decouple the dynamics from the thermodynamics, in such a way that the mechanical energy (the sum of kinetic energy (KE) and gravitational potential energy (GPE)) and the internal energy (IE) obey independent conservation laws.

The coupling between dynamics and thermodynamics is reinstated, however, when the Boussinesq and anelastic approximations are appended with a representation of irreversible processes due to molecular viscous and diffusive processes, and/or when the nonlinearities of the equation of state are no longer neglected. Such an approach is used for instance in the field of numerical ocean modelling. The way the coupling between the dynamics and thermodynamics manifests itself in that case is through the appearance of nonconservative terms in the local balance equation for the mechanical energy, which indicates the need for conversions with internal energy if total energy is to be conserved. Whereas the approximate forms of KE and GPE are close to their nonapproximated forms, the approximate form of IE has remained so far very mysterious, given that only the conversion terms with IE are explicitly represented in the Boussinesq and anelastic approximations, not IE itself. Since the approximate form of IE is in general unknown and left implicit, it is in general not straightforward to determine 
whether the approximation obtained by adding viscous and diffusive terms has a well-defined energy budget. Moreover, understanding the role of IE in such approximations may give rise to misinterpretations as in the case discussed by Tailleux [1].

The lack of explicit knowledge about the form assumed by the internal energy in the Boussinesq and anelastic approximations makes it difficult to understand the precise role played by internal energy (and hence the dynamics/ thermodynamics coupling) in incompressible or nearly incompressible turbulent stratified fluid flows. Until now, this difficulty has been systematically avoided by assuming the conversions to and from internal energy, and hence the dynamics/thermodynamics coupling, to be dynamically unimportant at leading order. Recently, however, Tailleux [1] challenged this assumption by showing from first principles that it was inconsistent with the second law, by suggesting that the coupling between dynamics and thermodynamics should increase both with the strength of the stratification and the degree of turbulence in the fluid.

The main objective of this paper will be to provide insights into the dynamics/thermodynamics coupling in weakly compressible turbulent stratified fluid flows. In particular, it will serve as follows.

(1) It will provide a general overview of the fundamental physical and technical issues involved in understanding the coupling between dynamics and thermodynamics in turbulent stratified fluids. It will explain the importance of isolating the part of the total potential energy (gravitational + potential) that is available for reversible conversions into kinetic energy, as well as the part of the internal energy available for irreversible conversions into mechanical energy. This is based on the results by Tailleux $[1,2]$.

(2) It will show how to construct explicitly the full range of known thermodynamic potentials, including entropy, internal energy, enthalpy, that have previously remained implicit in the Boussinesq and anelastic approximations. It will show how it is possible to establish a formal correspondence between the work of expansion/contraction in compressible fluids, and the changes in gravitational potential energy in the Boussinesq and anelastic approximations.

(3) It will show how the above results can help clarify and provide new insights into the energetics of turbulent mixing in turbulent stratified fluid flows, as discussed in Tailleux [1].

(4) It will discuss the general issue of how to estimate the overall work of expansion/contraction in steady-state buoyancy driven circulations, which has received much attention over the past decade in relation with understanding the relative importance of the surface buoyancy fluxes in driving and stirring the ocean circulation.

\section{Energetics and Dynamics/Thermodynamics Coupling in Compressible Fluids}

2.1. Compressible Navier-Stokes Equations for a Binary Fluid. Issues pertaining to the coupling between the dynamics and thermodynamics in turbulent-stratified fluids are fundamentally rooted in the description of the energetics of a fully compressible fluid. For most fluids of interest, this can be achieved in the context of the compressible Navier-Stokes equations. To fix ideas, these equations are given here for a binary fluid with a nonlinear equation of state such as seawater, namely,

$$
\begin{gathered}
\rho \frac{D \mathbf{v}}{D t}+\nabla P=-\rho \nabla \Phi+\nabla \cdot \mathbf{S} \\
\frac{D \rho}{D t}+\rho \nabla \cdot \mathbf{v}=0 \\
\rho \frac{D \eta}{D t}=\frac{\rho \dot{q}}{T}=-\nabla \cdot\left(\rho \mathbf{F}_{\eta}\right)+\rho \dot{\eta}_{\text {irr }}, \\
\rho \frac{D S}{D t}=-\nabla \cdot\left(\rho \mathbf{F}_{S}\right), \\
P=P(\eta, S, v)=-\frac{\partial e}{\partial v}, \quad T=T(\eta, S, v)=\frac{\partial e}{\partial \eta}
\end{gathered}
$$

where $\mathbf{v}=(u, v, w)$ is the three-dimensional velocity field, $\rho$ is the density, $P$ is the pressure, $\Phi=g_{0} Z$ is the geopotential expressed in terms of a constant gravitational acceleration $g_{0}$ and geopotential height $Z=Z(z)$ and $z$ the geometrical height, $\mathbf{S}$ is the deviatoric stress tensor, $\eta$ is the specific entropy, $S$ is salinity, $e=e(\eta, S, v)$ is the specific internal energy regarded as a function of entropy, salinity, and specific volume $v=1 / \rho, T$ is the temperature, $\kappa$ is the molecular diffusivity, $c_{p}$ is the specific heat at constant pressure, $\varepsilon_{K}$ is the viscous dissipation rate, $\mathbf{F}_{\eta}$ and $\mathbf{F}_{S}$ are molecular diffusive fluxes of entropy and salt, while $\dot{\eta}_{\text {irr }}>0$ is the irreversible production of entropy imposed by the second law of thermodynamics. In practice, these equations need to be supplemented with appropriate boundary conditions, for which specific examples will be discussed in the text.

2.2. Energetics and Dynamics/Thermodynamics Coupling. In order to discuss the nature of the coupling between dynamics and thermodynamics for a compressible fluid, we first form a local evolution equation for the mechanical energy $E_{m}=$ $\mathbf{v}^{2} / 2+\Phi$, that is, the sum of kinetic energy and gravitational potential energy, by multiplying the momentum equation (1); after some rearrangement, one thus obtains

$$
\rho \frac{D}{D t}\left[\frac{\mathbf{v}^{2}}{2}+\Phi\right]+\nabla \cdot\left(P \mathbf{v}-\rho \mathbf{F}_{k e}\right)=\rho P \frac{D v}{D t}-\rho \varepsilon_{K},
$$

which can also be rewritten in conservative form as follows:

$$
\frac{\partial\left(\rho E_{m}\right)}{\partial t}+\nabla \cdot\left[\rho\left(E_{m}+\frac{P}{\rho}\right) \mathbf{v}-\rho \mathbf{F}_{k e}\right]=\rho P \frac{D v}{D t}-\rho \varepsilon_{K},
$$

where the work against the stress tensor $\mathbf{v} \cdot \nabla \cdot \mathbf{S}=\nabla \cdot\left(\rho \mathbf{F}_{k e}\right)-$ $\rho \varepsilon_{K}$ has been decomposed into the divergence of the viscous 
flux of kinetic energy minus the viscous dissipation rate $\rho \varepsilon_{K}$. Physically, the presence of the right-hand side in both (6) and (7) indicates that mechanical energy $E_{m}$ is not a conservative quantity. The principle of total energy conservation suggests that the r.h.s. must therefore represent a conversion with internal energy.

In order to make this apparent, we now turn to the derivation of a local evolution equation for the internal energy. From the well-known expression of its total differential form: $\mathrm{d} e=T \mathrm{~d} \eta+\mu \mathrm{d} S-P \mathrm{~d} v$, combined with the evolution equations for entropy and salt (3) and (4), it follows that

$$
\begin{aligned}
\rho \frac{D e}{D t}= & \rho\left[T \frac{D \eta}{D t}+\mu \frac{D S}{D t}-P \frac{D v}{D t}\right]=T\left[-\nabla \cdot\left(\rho \mathbf{F}_{\eta}\right)+\rho \dot{\eta}_{\text {irr }}\right] \\
& -\mu \cdot \nabla \cdot\left(\rho \mathbf{F}_{S}\right)-\rho P \frac{D v}{D t} \\
= & -\nabla \cdot\left[\rho\left(T \mathbf{F}_{\eta}+\mu \mathbf{F}_{S}\right)\right] \\
& +\rho\left[\mathbf{F}_{\eta} \cdot \nabla T+\mathbf{F}_{S} \cdot \nabla \mu+T \dot{\eta}_{\text {irr }}\right]-\rho P \frac{D v}{D t} .
\end{aligned}
$$

By summing the mechanical energy equation with the internal energy, we arrive at the following result:

$$
\begin{array}{r}
\frac{\partial(\rho E)}{\partial t}+\nabla \cdot\left[\rho\left(E+\frac{P}{\rho}\right) \mathbf{v}-\rho \mathbf{F}_{k e}+\rho \mathbf{F}_{q}\right] \\
=+\rho \underbrace{\left[\mathbf{F}_{\eta} \cdot \nabla T+\mathbf{F}_{S} \cdot \nabla \mu+T \dot{\eta}_{\mathrm{irr}}-\varepsilon_{K}\right]}_{\dot{E}_{\mathrm{irr}}},
\end{array}
$$

where $E=E_{m}+e$ is the total energy, while we also defined $\mathbf{F}_{q}=T \mathbf{F}_{\eta}+\mu \mathbf{F}_{S}$ as a generalised heat flux. This in turn provides the expression for the entropy flux $\mathbf{F}_{\eta}=\left(\mathbf{F}_{q}-\mu \mathbf{F}_{S}\right) / T$. Now, the principle of total energy conservation implies that the total energy within a control volume can only change through energy and mass fluxes through this control volume. This in turn requires that the term $\dot{E}_{\text {irr }}$ vanishes identically, which imposes the following constraint on the irreversible entropy production term:

$$
\dot{\eta}_{\text {irr }}=\frac{\varepsilon_{K}-\mathbf{F}_{\eta} \cdot \nabla T-\mathbf{F}_{S} \cdot \nabla \mu}{T} .
$$

This in turn implies constraints on the admissible forms of the entropy flux $\mathbf{F}_{\eta}$ and salt flux $\mathbf{F}_{S}$, which need to be such that $\dot{\eta}_{\text {irr }} \geq 0$ in all circumstances. We also see that the irreversible production of entropy must vanish at thermodynamic equilibrium, which for a binary fluid is achieved when $T$ and $\mu$ become uniform. This is clearly the case here. It follows from these considerations that the internal energy equation can therefore be rewritten as

$$
\rho \frac{D e}{D t}+\nabla \cdot\left(\rho \mathbf{F}_{q}\right)=-\rho P \frac{D v}{D t}+\rho \varepsilon_{K}
$$

Note that this expression is consistent with the classical form of the first law of thermodynamics $D e / D t=\dot{q}+\dot{w}$, with $\dot{q}=\varepsilon_{K}-\rho^{-1} \nabla \cdot\left(\rho \mathbf{F}_{q}\right)$ as the local rate of heat transfer, and $\dot{w}=-P D v / D t$ as the local rate of work transfer. According to (11), the coupling between the dynamics and thermodynamics can be regarded as the sum of two effects. The first effect is due to the viscous dissipation of kinetic energy into the "heat" part of internal energy, while the second is related to the conversion between the elastic part of internal energy and kinetic energy. It is the latter term that is usually assumed to be of secondary importance in weakly compressible turbulent stratified fluids at low Mach number, and therefore the one under focus in this paper.

2.3. Energetics of Purely Buoyancy-Driven Circulations. As further motivation and justification for investigating the coupling between the dynamics and thermodynamics in turbulent-stratified fluids, let us simply point out that the work of expansion/contraction plays a central role in the energetics of purely buoyancy-driven circulations, as in horizontal convection, for example, Hughes and Griffiths [3], which has received much attention in the oceanography community over the past ten years. Thus, for a fluid heated and cooled at the top, assumed to be a surface of constant geopotential, the mechanical energy balance is as follows:

$$
\underbrace{\int_{V} P \frac{D v}{D t} \mathrm{~d} m}_{B}=\int_{V} \rho \varepsilon_{K} \mathrm{~d} V,
$$

where $\mathrm{d} m=\rho \mathrm{d} V$ is the mass of an elementary fluid element, and expresses the fact that in a steady state, the work of expansion/contraction must balance the volume-integrated viscous dissipation. Such a balance was recently discussed in details by Tailleux [2] in the context of the "ocean heat engine controversy." The importance of understanding what such a balance tells us about $B$ and the viscous dissipation stems from the central role played by $\varepsilon_{K}$ in the theory of turbulent flows, since it is the primary quantity entering Kolmogorov theory of turbulence for instance. The riddle here comes from the apparent conflict between the traditional belief that compressibility effects are small in weakly compressible fluids such as water and seawater, which on the basis of (12) would tend to suggest that flows in such fluids cannot really be large or be associated with significant amount of viscous dissipation, and the observation that even in water, buoyancy-driven flows can be sometimes vigorous, as in Rayleigh-Bénard convection, for instance, for which (12) is also supposed to hold. The primary purpose of this paper is to convince the reader that compressible effects in turbulent stratified fluids are significantly more important than usually assumed, and that such large compressible effects are actually described both by the incompressible Boussinesq and Anelastic approximations, in contrast to widely held beliefs to the contrary.

\section{Dynamics/Thermodynamics Coupling in Boussinesq/Anelastic Fluids}

3.1. Thermodynamically Consistent Boussinesq/Anelastic Approximations. Two main classes of "sound-proof" approximations are commonly used for the study of weakly 
compressible fluid flows at low Mach numbers, such as those typical of oceanic and atmospheric flows. These are the Boussinesq approximation, for example, Boussinesq [4], and the anelastic approximation, for example, Ogura [5]. As shown recently by Pauluis [6], improving on earlier ideas by Ingersoll [7], it is possible to derive a thermodynamically and energetically consistent set of anelastic equations for a binary fluid with an arbitrary nonlinear equation of state that also includes the Boussinesq approximation as a particular case. For the present purposes, these can be written as follows:

$$
\begin{aligned}
& \frac{D \mathbf{v}}{D t}+\nabla \cdot\left(\frac{\delta P}{\rho_{0}}\right)=-\left(\frac{\rho-\rho_{0}}{\rho_{0}}\right) \nabla \Phi+\frac{1}{\rho_{0}} \nabla \cdot \mathbf{S}, \\
& \nabla \cdot\left(\rho_{0} \mathbf{v}\right)=0 \\
& \rho_{0} \frac{D \eta}{D t}=\frac{\rho \dot{q}}{T}=-\nabla \cdot\left(\rho_{0} \mathbf{F}_{\eta}\right)+\dot{\eta}_{\mathrm{irr}} \\
& \rho_{0} \frac{D S}{D t}=-\nabla \cdot\left(\rho_{0} \mathbf{F}_{S}\right),
\end{aligned}
$$

where $\mathbf{v}=(u, v, w)$ is the three-dimensional velocity field, $\delta P=P-P_{0}$ is the difference between the total pressure $P$ and the reference pressure $P_{0}, \rho$ and $\rho_{0}$ are the density and reference density respectively, $\Phi=g_{0} Z$ is the geopotential, which can be written as the product of a constant gravitational acceleration times the geopotential height, a function of depth alone; $\mathbf{S}$ is the stress tensor, $\varepsilon_{K}$ is the viscous dissipation rate, $\eta$ is the specific entropy, and $S$ is the salinity. Pauluis [6] regards the second equation as one for the total water content. The present results are applicable to an arbitrary equation of state for a binary fluid in principle.

\subsection{Proof of Energetic and Thermodynamic Consistency. Prior} to the studies by Ingersoll [7], Pauluis [6], Young [8], Nycander [9], and Tailleux [10], it was generally thought to be impossible to derive energetically and thermodynamically consistent Boussinesq and anelastic approximations for a binary fluid with an arbitrary nonlinear equation of state. It is therefore of interest to verify such consistency, as it is closely related to understanding the nature of the dynamics/thermodynamics coupling in the BA system. To that end, we first derive the kinetic energy equation similarly as for the compressible case addressed above, which yields

$$
\rho_{0} \frac{D}{D t} \frac{\mathbf{v}^{2}}{2}+\nabla \cdot\left[\delta P \mathbf{v}-\rho_{0} \mathbf{F}_{k e}\right]=\rho_{0} b \frac{D Z}{D t}-\rho_{0} \varepsilon_{K},
$$

where $b=-g_{0}\left(\rho-\rho_{0}\right) / \rho_{0}$ is the buoyancy, and where $\mathbf{v} \cdot \nabla$. $\mathbf{S}=\nabla \cdot\left(\rho_{0} \mathbf{F}_{k e}\right)-\rho_{0} \varepsilon_{K}$ was split into a divergent term, and the classical viscous dissipation term, as previously.

The previous approaches to establishing the energetic consistency of the BA system by Ingersoll [7], Pauluis [6], Young [8], and Nycander [9] all similarly relied on linking the term $\rho_{0} b D Z / D t$ to the following function:

$$
h^{\ddagger}(\eta, S, Z)=h_{0}(\eta, S)-\int_{Z_{0}}^{Z} b\left(\eta, S, Z^{\prime}\right) \mathrm{d} Z,
$$

where $h_{0}(\eta, S)=h^{\ddagger}\left(\eta, S, Z_{0}\right)$ is a priori an arbitrary function of the adiabatically conserved variables $\eta$ and $S$. The evolution equation for such a function is, therefore,

$$
\rho_{0} \frac{D h^{\ddagger}}{D t}=\rho_{0} C_{\eta} \frac{D \eta}{D t}+\rho_{0} C_{S} \frac{D S}{D t}-\rho_{0} b \frac{D Z}{D t},
$$

where

$$
\begin{aligned}
C_{\eta} & =\frac{\partial h_{0}}{\partial \eta}-\int_{Z_{0}}^{Z} \frac{\partial b}{\partial \eta}\left(\eta, S, Z^{\prime}\right) \mathrm{d} Z^{\prime}, \\
C_{S} & =\frac{\partial h_{0}}{\partial S}-\int_{Z_{0}}^{Z} \frac{\partial b}{\partial S}\left(\eta, S, Z^{\prime}\right) \mathrm{d} Z^{\prime}
\end{aligned}
$$

Now, combining the kinetic energy equation with (19) leads to:

$$
\begin{gathered}
\rho_{0} \frac{D}{D t}\left[\frac{\mathrm{v}^{2}}{2}+h^{\ddagger}\right]+\nabla \cdot\left[\delta P \mathbf{v}-\rho_{0} \mathbf{F}_{k e}\right] \\
=\rho_{0}\left[C_{\eta} \frac{D \eta}{D t}+C_{S} \frac{D S}{D t}-\varepsilon_{K}\right] .
\end{gathered}
$$

This is an important result, which clearly shows that the energy quantity $\rho_{0}\left(\mathbf{v}^{2} / 2+h^{\ddagger}\right)$ is conserved in absence of viscous $\left(\varepsilon_{K}=0\right)$ and diabatic effects $(D \eta / D t=D S / D t=0)$ and hence can be regarded as the relevant energy of the BA system in such conditions. As argued by Tailleux [10], however, it is important to realize that the principle of energy conservation must hold both for adiabatic/inviscid conditions, as well as for diabatic/viscous ones. In the latter case, the r.h.s. of (21) will not vanish in general, which calls for the existence of an additional energy quantity $h^{*}$ satisfying an evolution equation of the type:

$$
\rho_{0} \frac{D h^{*}}{D t}+\nabla \cdot\left(\rho_{0} \mathbf{F}_{h}\right)=-\rho_{0}\left[C_{\eta} \frac{D \eta}{D t}+C_{S} \frac{D S}{D t}-\varepsilon_{K}\right],
$$

where the r.h.s. of (22) exactly cancels out the r.h.s. of (21), making the quantity $\mathbf{v}^{2}+h^{\ddagger}+h^{*}$ the relevant total energy for the BA system, rather than the quantity $\mathbf{v}^{2} / 2+h^{*}$. The problem of the latter approach, however, is that it focuses on the coupling between the adiabatic energy $\mathbf{v}^{2}+h^{\ddagger}$ and the diabatic energy $h^{*}$, which because of the function $h_{0}(\eta, S)$ does not appear to be uniquely defined. As a result, this makes it difficult to relate this way of discussing the energetics of the BA system to the classical approach.

Tailleux [10] showed that the energetic and thermodynamic consistency of the BA system can in fact be approached in a way closer to the classical approach by rewriting the kinetic energy equation as follows:

$$
\begin{gathered}
\rho_{0} \frac{D}{D t}\left(\frac{\mathbf{v}^{2}}{2}-b\left(Z-Z_{0}\right)\right)+\nabla \cdot\left[\delta P \mathbf{v}-\rho_{0} \mathbf{F}_{k e}\right] \\
\quad=-\rho_{0}\left(Z-Z_{0}\right) \frac{D b}{D t}-\rho_{0} \varepsilon_{K}
\end{gathered}
$$


by using the result that $b \mathrm{~d} Z=\mathrm{d}\left[b\left(Z-Z_{0}\right)\right]-\left(Z-Z_{0}\right) \mathrm{d} b$, which in conservative form becomes

$$
\begin{gathered}
\frac{\partial\left(\rho_{0} E_{m}\right)}{\partial t}+\nabla \cdot\left[\rho_{0}\left(E_{m}+\frac{\delta P}{\rho_{0}}\right) \mathbf{v}-\rho_{0} \mathbf{F}_{k e}\right] \\
=-\rho_{0}\left(Z-Z_{0}\right) \frac{D b}{D t}-\rho_{0} \varepsilon_{K}
\end{gathered}
$$

by defining the total mechanical energy by $\rho_{0} E_{m}=\rho_{0} \mathbf{v}^{2} / 2-$ $\rho_{0} b\left(Z-Z_{0}\right)=\rho_{0} \mathbf{v}^{2} / 2+g_{0}\left(\rho-\rho_{0}\right)\left(Z-Z_{0}\right)$, which is consistent with the traditional definition. Now, it is easily seen that (23) and (24) are directly comparable with the compressible mechanical energy equations (6) and (7) provided that it is meaningful to establish the following correspondence:

$$
\rho P \frac{D v}{D t} \longleftrightarrow-\rho_{0}\left(Z-Z_{0}\right) \frac{D b}{D t} .
$$

Tailleux [10] shows that such a correspondence is legitimate. In the Boussinesq case ( $\rho_{0}=$ constant), this can be shown by expanding the pressure and specific volume as follows: $P=$ $P_{a}-\rho_{0} g_{0} Z+\cdots$ and $v=1 / \rho=1 / \rho_{0}-\left(\rho-\rho_{0}\right) / \rho_{0}^{2}+\cdots$, so that

$\rho P \frac{D v}{D t} \approx-\rho_{0}\left(P_{a}-\rho_{0} g_{0} Z\right) \frac{D}{D t}\left(\frac{\rho-\rho_{0}}{\rho_{0}^{2}}\right)=-\rho_{0}\left(Z-Z_{0}\right) \frac{D b}{D t}$

in agreement with (25), provided that one defines $Z_{0}$ by $Z_{0}=$ $P_{a} /\left(\rho_{0} g_{0}\right)$. Although the anelastic case is somewhat more involved, it turns out that such a correspondence can also be established in the anelastic case, as discussed by Tailleux [10].

3.3. Thermodynamics of Boussinesq and Anelastic Fluids. The possibility to regard the term $\delta W_{b a}=\left(Z-Z_{0}\right) \mathrm{d} b$ as the counterpart of the compressible work $\delta W=-P \mathrm{~d} v$ is an important result, because it naturally points to the method by which to construct the thermodynamics of the Boussinesq/Anelastic system. Indeed, let us recall that a basic tenet of classical thermodynamics is that the specific internal energy $e=e(\eta, S, v)$, whose total differential is given by

$$
\mathrm{d} e=T \mathrm{~d} \eta+\mu \mathrm{d} S-P \mathrm{~d} v,
$$

where $\mu$ is the relative chemical potential of seawater in seawater, is a function of state independent of the thermodynamic path followed. As a result, it follows that the generalised heat transfer differential $\delta Q=T \mathrm{~d} \eta+\mu \mathrm{d} S$ is not independent of the work transfer $\delta W=-P \mathrm{~d} v$. In classical thermodynamics, this interdependence is imposed by the socalled Maxwell relationships, for example, Callen [11], which simply expresses the result that the cross-derivatives with respect to two different variables must be equal for twice continuously differentiable functions. In the present case, the assumption that $e$ is a function of the thermodynamic state only implies that

$$
T=\frac{\partial e}{\partial \eta}, \quad \mu=\frac{\partial e}{\partial S}, \quad P=-\frac{\partial e}{\partial v},
$$

which in turn implies the following three Maxwell relationships:

$$
\begin{gathered}
\frac{\partial T}{\partial v}=\frac{\partial^{2} e}{\partial \eta \partial v}=-\frac{\partial P}{\partial \eta}, \quad \frac{\partial T}{\partial S}=\frac{\partial^{2} e}{\partial \eta \partial S}=\frac{\partial \mu}{\partial \eta}, \\
\frac{\partial \mu}{\partial v}=\frac{\partial^{2} e}{\partial S \partial v}=-\frac{\partial P}{\partial S} .
\end{gathered}
$$

The above remarks suggest that the simplest way to ensure that the BA system is energetically and thermodynamically consistent is to ensure that the approximation to the generalised heat transfer, denoted here by $\delta Q_{b a}=T_{b a} \mathrm{~d} \eta_{b a}+\mu_{b a} \mathrm{~d} S_{b a}$, is similarly linked via relevant Maxwell relationships to the approximation to the work transfer identified previously, namely,

$$
\delta W_{b a}=\left(Z-Z_{0}\right) \mathrm{d} b,
$$

where the subscript $b a$ was used to denote the approximation to the generalised "heat variables and functions" $T, \eta, \mu$, and $S$. If so, this would in turn allows one to regard the following expression:

$$
\mathrm{d} e_{b a}=T_{b a} \mathrm{~d} \eta+\mu_{b a} \mathrm{~d} S+\left(Z-Z_{0}\right) \mathrm{d} b,
$$

as the natural counterpart of the fundamental relation of thermodynamics (27), and hence as the total differential of the relevant approximation to the internal energy of the "BA fluid," for which the natural variables are entropy $\eta$, salinity $S$, and buoyancy $b$. In practice, however, it is often more convenient to work with pressure $P$ as a dependent variable rather than specific volume, as well as with temperature $T$ rather than entropy $\eta$, which motivates the introduction of additional thermodynamic potentials constructed from (27) by means of the Legendre transform, for example, Alberty [12]. The most common thermodynamic potentials that are also the most relevant for the present work are the specific enthalpy $h=e+p v$ and the Gibbs free energy $g=e+p v-T \eta$, whose natural dependent variables are $(\eta, S, P)$ and $(T, S, P)$, respectively. From (31), it is easy to convince oneself that the corresponding approximations to $h$ and $g$ are given by $h_{b a}=e_{b a}-b\left(Z-Z_{0}\right)$ and $g_{b a}=e_{b a}-b\left(Z-Z_{0}\right)-T_{b a} \eta_{b a}$, with the following total differentials:

$$
\begin{aligned}
\mathrm{d} h_{b a} & =d\left[e_{b a}-b\left(Z-Z_{0}\right)\right] \\
& =T_{b a} \mathrm{~d} \eta_{b a}+\mu_{b a} \mathrm{~d} S_{b a}-b \mathrm{~d} Z \\
\mathrm{~d} g_{b a} & =d\left[e_{b a}-b\left(Z-Z_{0}\right)-T_{b a} \eta_{b a}\right] \\
& =-\eta_{b a} \mathrm{~d} T_{b a}+\mu_{b a} \mathrm{~d} S_{b a}-b \mathrm{~d} Z .
\end{aligned}
$$

Until now, the above considerations have remained rather formal, and while they indicate that it is in principle possible to construct the full range of known thermodynamic potentials for a BA fluid, they have not addressed the issue of how such potentials might be constructed in practice.

In the previous approaches by Ingersoll [7], Pauluis [6], Young [8], and Nycander [9], the authors have generally assumed the buoyancy $b=b(\eta, S, Z)$ to be given as a function 
of the dependent variables considered, typically entropy, salinity, and geopotential height $Z$. From (32), this can be integrated with respect to $Z$, namely,

$$
h_{b a}(\eta, S, Z)=h_{b a}\left(\eta, S, Z_{0}\right)-\int_{Z_{0}}^{Z} b\left(\eta, S, Z^{\prime}\right) \mathrm{d} Z^{\prime},
$$

for some reference geopotential height $Z_{0}$, traditionally taken at the ocean surface in the oceanic case, but this only provides an expression for the specific enthalpy up to an indeterminate function of entropy and salinity, which cannot be specified without additional thermodynamic information about the fluid. This problem arises simply because the knowledge of density alone is in general never sufficient by itself to specify all possible thermodynamic properties of the fluid, which in general also requires some knowledge of such properties as the heat capacity, speed of sound, for example, see Feistel [13] or Callen [11] for exhaustive discussion of this issue. For this reason, it seems preferable to investigate the possibility of deriving the approximate thermodynamic potentials from the knowledge of the Gibbs function $g=$ $g(T, S, P)$, for instance, which is currently used to synthesise all thermodynamic properties for seawater as discussed in Feistel [13]. Here, we show that the function,

$$
g_{b a}=g_{b a}(T, S, Z)=g\left(T, S, P_{0}(Z)\right)+g_{0}\left(Z-Z_{0}\right),
$$

satisfies the above differential relationships and hence can be regarded as the relevant Boussinesq/Anelastic approximation to the Gibbs function. Indeed, from the result that $\mathrm{d} g=$ $-\eta \mathrm{d} T+\mu \mathrm{d} S+v \mathrm{~d} P$, it follows that the total differential of $g_{b a}$ is given by

$$
\mathrm{d} g_{b a}=-\tilde{\eta} \mathrm{d} T+\tilde{\mu} \mathrm{d} S+g_{0}\left(1-\rho_{0} \widetilde{v}\right) \mathrm{d} Z,
$$

where we used $\mathrm{d} P_{0}=-\rho_{0} g_{0} \mathrm{~d} Z$, and where the tilde quantities denote the exact quantities in which the actual pressure is replaced by the reference pressure $P_{0}(Z)$, that is, $\tilde{\eta}=$ $\eta\left(T, S, P_{0}(Z)\right), \tilde{\mu}=\mu\left(T, S, P_{0}(Z)\right)$, and $\widetilde{v}=v\left(T, S, P_{0}(Z)\right)$. This expression is consistent with the previous one, provided that one chooses

$$
\begin{gathered}
\eta_{b a}=\tilde{\eta}, \quad \mu_{b a}=\tilde{\mu}, \\
b=-g_{0}\left(1-\rho_{0} \tilde{v}\right)=-g_{0}\left(\frac{\tilde{\rho}-\rho_{0}}{\tilde{\rho}}\right) .
\end{gathered}
$$

This in turn shows that the density entering the definition of the buoyancy is actually related to the "true" density by $\rho=\rho_{0}+\rho_{0} / \tilde{\rho}\left(\tilde{\rho}-\rho_{0}\right)=2 \rho_{0}-\rho_{0}^{2} / \tilde{\rho}$.

Assuming that $g$ is known, we can deduce the following thermodynamic potentials as follows:

$$
\begin{aligned}
e_{b a}(S, T, Z) & =g_{b a}+b\left(Z-Z_{0}\right)+T_{b a} \eta_{b a} \\
& =\tilde{g}+\left(g_{0}+b\right)\left(Z-Z_{0}\right)-T \frac{\partial \tilde{g}}{\partial T}, \\
h_{b a}(S, T, Z) & =e_{b a}-b\left(Z-Z_{0}\right)=\tilde{g}+g_{0}\left(Z-Z_{0}\right)-T \frac{\partial \tilde{g}}{\partial T},
\end{aligned}
$$

which are, respectively, associated with the total differentials:

$$
\begin{gathered}
\mathrm{d} e_{b a}=T \mathrm{~d} \tilde{\eta}+\tilde{\mu} \mathrm{d} S+\left(Z-Z_{0}\right) \mathrm{d} b, \\
\mathrm{~d} h_{b a}=T \mathrm{~d} \tilde{\eta}+\tilde{\mu} \mathrm{d} S-b \mathrm{~d} Z .
\end{gathered}
$$

While the above approach is well suited to the case of seawater whose Gibbs function is now well documented, it is arguably less relevant to the case of a fluid whose thermodynamic properties have been synthesised using a different kind of thermodynamic potential. In theory, such a synthesis can indeed be achieved in many ways, for instance, using internal energy, enthalpy, Helmholtz-free energy, or even the more esoteric Massieu functions as discussed by Callen [11]. While it is beyond the scope of this paper to treat all possible cases, it is useful for illustration to discuss the particular case where the specific enthalpy $h=h(\eta, S, P)$ is the thermodynamic potential used to synthesise the thermodynamic properties of the fluid considered. In that case, it is left to the reader to check that the relevant approximation $h_{b a}$ to the specific enthalpy $h$ is then given by $h_{b a}=h\left(\eta, S, P_{0}(Z)\right)+g_{0}\left(Z-Z_{0}\right)$, as can be verified from taking the total differential of the latter expression, namely,

$$
\mathrm{d} h_{b a}=\widetilde{T} \mathrm{~d} \eta+\tilde{\mu} \mathrm{d} S+g_{0}\left(1-\rho_{0} g \widetilde{v}\right) \mathrm{d} Z=\widetilde{T} \mathrm{~d} \eta+\tilde{\mu} \mathrm{d} S-b \mathrm{~d} Z,
$$

which is in agreement with (32). As seen previously, $e_{b a}=$ $h_{b a}+b\left(Z-Z_{0}\right)$, which implies that the the internal energy can then be written as $e_{b a}=\widetilde{h}+\left(g_{0}+b\right)\left(Z-Z_{0}\right)=\widetilde{h}+$ $\rho_{0} g_{0}\left(Z-Z_{0}\right) \widetilde{v}$.

The above derivations therefore make it clear that it is possible to use any existing knowledge of the thermodynamic properties of the fluid considered to reconstruct the relevant approximations for a BA fluid, and also that the approximations underlying the construction of the BA system of equations only slightly alter the form of the thermodynamic properties, which remain close to that of a fully compressible fluid.

\section{Consequence for Understanding the Energetics of Turbulent Stratified Fluids}

4.1. Model Formulation. In this section, we discuss the consequences of the above results for our understanding of the energetics of turbulent mixing in stratified fluids, which were originally pointed out by Tailleux [1]. Until recently, the energetics of turbulent-stratified mixing had been essentially discussed for an incompressible Boussinesq fluid with a linear equation of state governed by the following equations:

$$
\begin{gathered}
\frac{D \mathbf{v}}{D t}+\frac{1}{\rho_{0}} \nabla P=-\frac{\rho}{\rho_{0}} g_{0} \mathbf{k}+\nu \nabla^{2} \mathbf{v}, \\
\nabla \cdot \mathbf{v}=0, \\
\frac{D T}{D t}=\kappa \nabla^{2} T, \\
\rho=\rho_{0}\left[1-\alpha\left(T-T_{0}\right)\right],
\end{gathered}
$$

where $\alpha$ is the thermal expansion coefficient, $\nu$ the molecular viscosity, and $\kappa$ the molecular diffusivity, with all remaining 
notations as before. The situation considered is that of a freely decaying turbulent flow. For instance, a configuration extensively studied is that of a stably stratified parallel shear flow that becomes unstable. Turbulent behaviour ensues until the flow relaminarises, see Tailleux [1] for references. As a result of turbulence, diffusive mixing is enhanced. Here, mixing refers to the irreversible modification of temperature by molecular diffusion. Winters et al. [14] introduced a conceptual framework able to quantify precisely the amount of mixing taking place during a turbulent mixing event.

4.2. The Classical View of the Energetics of Turbulent Mixing. The classical view on the energetics of turbulent mixing has usually revolved on the idea that during a turbulent mixing event, although most of the initial kinetic energy of the parallel shear flow is lost to viscous dissipation as expected, a significant fraction (often reported to be around 20 percent) appears to be lost to gravitational potential energy. This can be mathematically formulated by considering the evolution equation for the kinetic energy, namely,

$$
\rho_{0} \frac{D}{D t} \frac{\mathbf{v}^{2}}{2}+\nabla \cdot\left(P \mathbf{v}-\rho_{0} \mathbf{F}_{k e}\right)=-\rho g w-\rho_{0} \varepsilon_{K}
$$

integrated over the volume of the fluid, as well as over the duration of a turbulent mixing event, which yields

$$
\Delta \mathrm{KE}=-\overline{\int_{V} \rho g w \mathrm{~d} V}-\overline{\int_{V} \rho_{0} \varepsilon_{K} \mathrm{~d} V}<0,
$$

where the overbar denotes the integration over the relevant time interval, and $\triangle \mathrm{KE}$ is the difference between the final and initial kinetic energy. Looking now at the evolution of the gravitational potential energy,

$$
\frac{D(\rho g z)}{D t}=\rho g w+g z \frac{D \rho}{D t}=\rho g w+g z \kappa \nabla^{2} \rho,
$$

integrating over the volume, as well as in time over the duration of the mixing event, we obtain

$$
\Delta \mathrm{GPE}=\overline{\int_{V} \rho g w \mathrm{~d} V}+\overline{\underbrace{\int_{V} g z \kappa \nabla^{2} \rho}_{W_{\text {laminar }}}} .
$$

According to the latter equation, the net change in gravitational potential energy $\triangle \mathrm{GPE}$ is made up of two contributions, one in appearance occurring at the expenses of the kinetic energy of the shear flow, and the other to due to internal energy via the term $W_{\text {laminar. }}$ In this description, the role of internal energy appears to be very limited and restricted to the weak laminar conversion $W_{\text {laminar }}$. On the other hand, a somewhat puzzling feature of the above description is that the term $\rho g w$ is supposed to represent in principle a reversible conversion of KE into GPE. Yet, the net change in GPE is irreversible by construction, as the time integration is achieved over a turbulent mixing event separated by laminar evolution. In other words, while reversible conversions of KE into GPE are physically well understood, irreversible conversion of KE into GPE is significantly harder to rationalise.
4.3. Available Potential Energy and Irreversible Mixing. As first recognised by Lorenz [15], only a small fraction of the total gravitational potential energy is generally available for reversible conversion into kinetic energy. This fraction is usually referred to as the "available potential energy" (APE) and is defined as the difference in potential energy between the actual state and the reference state of minimum potential energy obtained in an adiabatic rearrangement of the fluid parcels. Physically, it can be shown that the density $\rho_{r}(z, t)$ and pressure $P_{r}(z, t)$ of the reference state depend on $z$ and $t$ only. Moreover, they must be in hydrostatic equilibrium at all times, namely,

$$
\frac{\partial P_{r}}{\partial z}=-\rho_{r} g_{0}
$$

Physically, the reference state can be described in terms of the function $z_{r}=z_{r}(\mathbf{x}, t)$ to represent the position that a parcel located at $(\mathbf{x}, t)$ in the actual state would have in the reference state. As a result, the assumption that the parcel is displaced adiabatically from $(\mathbf{x}, t)$ to $z_{r}(\mathbf{x}, t)$ implies that $\rho_{r}\left(z_{r}, t\right)=\rho(\mathbf{x}, t)$, that is, the parcel retains its density in the adiabatic displacement. This makes it possible to derive the following expressions for the background gravitational potential energy and available gravitational potential energy:

$$
\begin{gathered}
\mathrm{GPE}_{r}=\int_{V} \rho_{r}\left(z_{r}\right) g_{0} z_{r} \mathrm{~d} V=\int_{V} \rho g_{0} z_{r} \mathrm{~d} V, \\
\mathrm{AGPE}=\int_{V} \rho g_{0}\left(z-z_{r}\right) \mathrm{d} V .
\end{gathered}
$$

Because the reference state is obtained from the actual state by an adiabatic transformation, it follows that it can only change with time if diabatic effects are present in the system. The work by Winters et al. [14] was the first study to suggest that such a property could be used as a rigorous way to diagnose the amount of irreversible mixing taking place in stably stratified turbulent fluids, and suggested to estimate the degree of turbulent mixing taking place in a fluid from the rate of change of $\mathrm{GPE}_{r}$. This idea can be put on a rigorous footing by deriving the following evolution equation for $\mathrm{GPE}_{r}$ :

$$
\begin{aligned}
\frac{\mathrm{dGPE}_{r}}{\mathrm{~d} t} & =\int_{V} \rho g_{0} \frac{D z_{r}}{D t} \mathrm{~d} V+\int_{V} g_{0} z_{r} \frac{D \rho}{D t} \mathrm{~d} V \\
& =\int_{V} g_{0} z_{r} \kappa \nabla^{2} \rho \mathrm{d} V .
\end{aligned}
$$

A key result in Winters et al. [14] was to show that the first term in the r.h.s. of (48) always vanishes, by using an explicit construction of the reference density profile. Since then, the result was generalised to a fully compressible fluid by Tailleux [1], while Pauluis [16] showed that the result follows naturally from the reference state being a constrained potential energy minimum. As a result, (48) becomes

$$
W_{r, \text { mixing }}=\int_{V} g_{0} z_{r} \kappa \nabla^{2} \rho \mathrm{d} V=-\int_{V} g_{0} \kappa \frac{\partial \rho_{r}}{\partial z_{r}}\left\|\nabla z_{r}\right\|^{2} \mathrm{~d} V>0,
$$


where the last part was obtained by using an integration by parts, assuming the density flux to vanish at all boundaries, and using the result that $\rho(\mathbf{x}, t)=\rho_{r}\left(z_{r}(\mathbf{x}, t), t\right)$ by construction, so that $\nabla \rho=\partial \rho_{r} / \partial z_{r} \nabla z_{r}$. The result that $W_{r, \text { mixing }}>$ 0 follows from the fact that $\partial \rho_{r} / \partial z_{r} \neq 0$ everywhere, since the reference state is stably stratified by definition. Equation

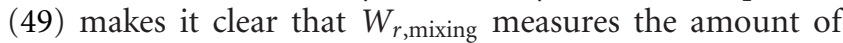
turbulent mixing taking place in the system, because $\left\|\nabla z_{r}\right\|^{2}$ measures the degree to which isopycnal surfaces are distorted by turbulent stirring. As a result, we can therefore write

$$
\begin{aligned}
\frac{\mathrm{dAGPE}}{\mathrm{d} t} & =\frac{\mathrm{d}\left(\mathrm{GPE}-\mathrm{GPE}_{r}\right)}{\mathrm{d} t} \\
& =\int_{V} \rho g_{0} w \mathrm{~d} V+W_{\text {laminar }}-W_{r, \text { mixing }}
\end{aligned}
$$

4.4. Energy Budget of a Turbulent Mixing Event. Let us now return to the problem of understanding the energetics of a turbulent mixing event, again defined as an episode of intense turbulent mixing characterised by potentially high values of AGPE preceded and followed by laminar conditions for which $A G P E=0$. Thus, integrating the above equations over the duration of one such turbulent mixing event yields

$$
\begin{gathered}
\Delta \mathrm{AGPE}=0=\overline{\int_{V} \rho g_{0} w \mathrm{~d} V}+\bar{W}_{\text {laminar }}-\bar{W}_{r, \text { mixing }}, \\
\Delta \mathrm{GPE}_{r}=\bar{W}_{r, \text { mixing }} .
\end{gathered}
$$

The first important conclusion that one may infer from (51) is that the buoyancy flux $\int_{V} \overline{\rho g w} \mathrm{~d} V$ can be linked to the time-and volume-integrated irreversible diabatic effects due to molecular diffusion since (51) implies

$$
\overline{\int_{V} \rho g_{0} w \mathrm{~d} V}=\bar{W}_{r, \text { mixing }}-\bar{W}_{\text {laminar }}=D(\mathrm{APE}),
$$

where $D(\mathrm{APE})$ represents the dissipation of AGPE by means of molecular diffusive processes. The second important conclusion inferred from (52) is that the total amount of turbulent mixing having taking place during the turbulent mixing event can be measured from the net change in $\mathrm{GPE}_{r}$. Regarding the latter equation, Winters et al. [14] suggested that its physical meaning is best understood by rewriting it as follows:

$$
\Delta \mathrm{GPE}_{r}=D(\mathrm{APE})+\bar{W}_{\text {laminar }},
$$

which suggests that the fraction of kinetic energy that is consumed by turbulent mixing but not dissipated by viscous friction is first converted reversibly into AGPE, but then irreversibly converted somehow into $\mathrm{GPE}_{r}$ by molecular diffusion, as illustrated in Panel (b) of Figure 1, with an additional small contribution to the net increase of $\mathrm{GPE}_{r}$ being due to internal energy.

4.5. Alternative Interpretation of the Energetics of Turbulent Mixing. It is important to remark that the validity of Winters et al. [14] interpretation relies on the idea that the irreversible conversion of one form of mechanical energy (AGPE) into another form of mechanical energy $\left(\mathrm{GPE}_{r}\right)$ is a physically legitimate one. As discussed by Tailleux [1], however, this idea is difficult to accept from the vantage point of thermodynamics, because the irreversible character of such a conversion necessarily implies that it should be accompanied by a change of entropy, and therefore of internal energy. Indeed, assuming the reverse to be true would lead to a contradiction. This is because for no change in internal energy to occur, an equal and opposite amount of compressible work $\delta W=$ $-P \mathrm{~d} v$ would be needed to exactly balance the heat transfer $\delta Q=T \mathrm{~d} \eta_{\text {irr }}$ implied by the assumed diabatic and irreversible character of the $\mathrm{AGPE} / \mathrm{GPE}_{r}$ conversion. However, this would violate the second law of thermodynamics, because it is well known from the theory of heat engines that only a small fraction of the heat transfer $\delta Q$ can be transformed into useful work $\delta W$, for example, Callen [11], which implies the impossibility for $\delta W$ and $\delta Q$ to be equal in magnitude.

The above difficulty is easily resolved, however, if one accepts to regard the term $\left(Z-Z_{0}\right) \mathrm{d} b$ as a conversion between internal energy and gravitational potential energy in the energetics of the BA system. Indeed, note that the term $W_{r \text {,mixing }}$ can be rewritten as follows:

$$
W_{r, \text { mixing }}=\int_{V} g_{0} z_{r} \frac{D \rho}{D t} \mathrm{~d} V=-\int_{V} \rho_{0} z_{r} \frac{D b}{D t} \mathrm{~d} V,
$$

which is precisely of the form associated with a conversion of internal energy into mechanical energy, with $z_{r}$ instead of $Z-Z_{0}$. If so, $W_{r \text {,mixing }}$ and $D(\mathrm{APE})$ should therefore be naturally regarded as conversions between internal energy and gravitational potential energy, suggesting that the internal energy should evolve according to

$$
\frac{\mathrm{d} I E}{\mathrm{~d} t}=D(\mathrm{KE})+D(\mathrm{APE})-W_{r, \text { mixing }} .
$$

Although such an interpretation now appears to be consistent with thermodynamics, the form of (56) suggests that it might still be possible for the APE dissipated into internal energy to be then converted into $\mathrm{GPE}_{r}$, in which case the whole argument would arguably look like a minor quibble, since it is hard to understand why it should matter whether $\mathrm{APE}$ is directly dissipated into $\mathrm{GPE}_{r}$ or via prior conversion into internal energy.

The latter issue was addressed by Tailleux [1], who argued that internal energy is best regarded as the sum of distinct types of internal energy, such that the kind of internal energy into which APE is dissipated differs from the kind of internal energy converted into $\mathrm{GPE}_{r}$, making it possible for (56) to hold without necessarily implying that the APE dissipated into internal energy is converted into $\mathrm{GPE}_{r}$. In particular, Tailleux [1] argue that in the same way that it is possible to decompose the temperature as follows:

$$
T(\mathbf{x}, t)=T_{0}(t)+T_{r}(z, t)+T^{\prime}(\mathbf{x}, t),
$$

that is, as the sum of a time-dependent mean temperature, a part accounting for the mean vertical gradient, and a residual, it is similarly possible to decompose the internal energy into three reservoirs:

$$
\mathrm{IE}=\mathrm{IE}_{0}+\mathrm{IE}_{\text {exergy }}+\mathrm{AIE},
$$




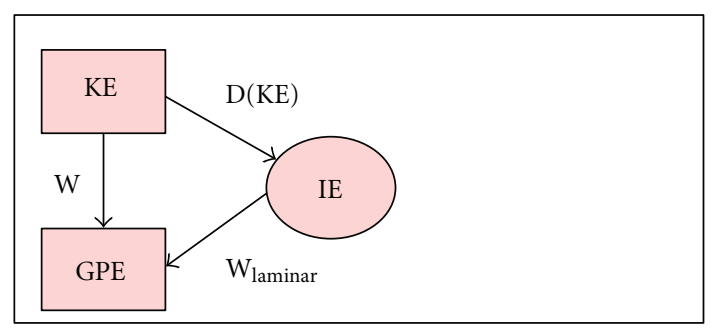

(a) Classical Energetics

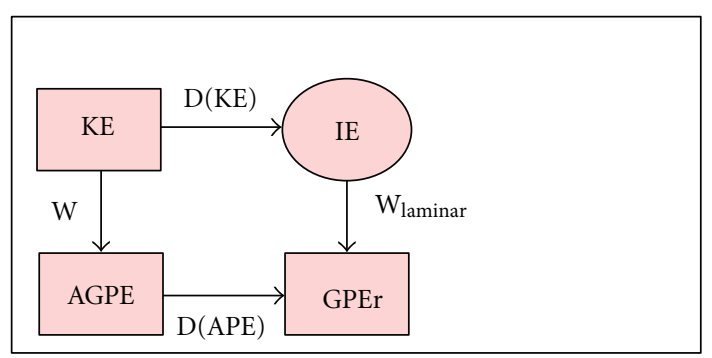

(b) Winters et al. [14]

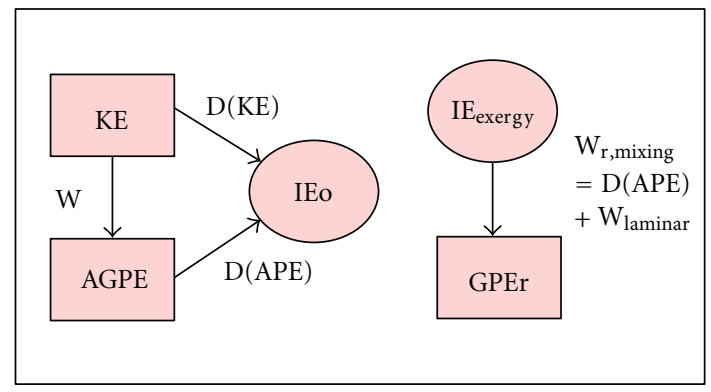

(c) Tailleux [1]

FIGURE 1: Evolution of ideas about the energetics of turbulent stratified mixing. (a) Interpretation seemingly following the result of classical energetics arguments based on the three reservoirs KE, GPE, and IE; (b) interpretation proposed by Winters et al. [14] based on the use of Lorenz [15] ideas to separate the gravitational potential energy into an available and unavailable component; (c) new interpretation proposed by Tailleux [1] on the basis of a rigorous analysis of the energetics of compressible Navier-Stokes equations aimed at elucidating the role of internal energy.

where $\mathrm{IE}_{0}$ is the internal energy of the thermodynamic equilibrium obtained from Lorenz [15] state in a process conserving the total enthalpy of the system, $\mathrm{IE}_{\text {exergy }}=\mathrm{IE}_{r}-\mathrm{IE}_{0}$ is the difference in internal energy between the internal energy of Lorenz [15] reference state, and $\mathrm{AIE}=\mathrm{IE}-\mathrm{IE}_{r}$ is the available internal energy, that is, the difference in internal energy between the actual state and Lorenz [15] reference state. Tailleux [1] showed how to derive evolution equations for each subcomponents of the internal energy and was able to show that at leading order, neglecting only very small terms, it is possible to decompose (56) into the two following equations for $\mathrm{IE}_{0}$ and $\mathrm{IE}_{\text {exergy }}$ as follows:

$$
\begin{aligned}
& \frac{\mathrm{dIE}_{0}}{\mathrm{~d} t} \approx D(\mathrm{KE})+D(\mathrm{APE}), \\
& \frac{\mathrm{dIE}_{\text {exergy }}}{\mathrm{d} t} \approx-W_{r, \text { mixing. }} .
\end{aligned}
$$

In physical terms, Tailleux [1] shows that increasing or decreasing $\mathrm{IE}_{0}$ is equivalent to increasing or decreasing the mean thermodynamic equilibrium temperature $T_{0}$, whereas decreasing $\mathrm{IE}_{\text {exergy }}$ is equivalent to smooth out the vertical temperature gradient of $T_{r}(z, t)$. As a result, (59) interpretation is that both the viscous dissipation of $\mathrm{KE}$ and the diffusive dissipation of APE increase $T_{0}$, whereas (60) interpretation is that turbulent mixing smoothes out the vertical gradient of $T_{r}(z, t)$. This is an important result, because both $W_{r \text {,mixing }}$ and $D(\mathrm{APE})$ are widely recognised to represent leading order energy conversions in turbulentstratified mixing. In the current dominant paradigm based on Winters et al. [14] interpretation, however, these conversions have been rationalised without invoking any significant role for the internal energy, in contrast to our interpretation. Physically, our interpretation also implies that whereas the dissipation of KE and APE should not be associated with any significant overall expansion of the fluid owing to its assumed weakly compressible nature, the release of exergy $\mathrm{IE}_{\text {exergy }}$ should in contrast be comparatively associated with a significant reduction in the volume of the fluid. By comparison, Winters et al. [14] interpretation call only for a tiny volume expansion due to the viscous dissipation, and for a tiny volume contraction due to the conversion of internal energy into $\mathrm{GPE}_{r}$ at the laminar rate $W_{\text {laminar. Figure } 2 \text { illus- }}$ trates a laboratory experiment that clearly tends to support our interpretation.

\section{Net Compressible Work in Purely Buoyancy-Driven Flows}

Having determined that the classical Boussinesq and anelastic approximations formally possess a representation of the coupling between the dynamics and thermodynamics that is comparable to that of the fully compressible Navier-Stokes equations, we now seek to illustrate some aspects of such coupling in the particular case of a purely buoyancy-driven circulation driven by differential heating/cooling imposed at the top boundary of the domain, as in the oceanic case, a configuration generally referred to as "horizontal convection," see Hughes and Griffiths [3] for a review. The importance 


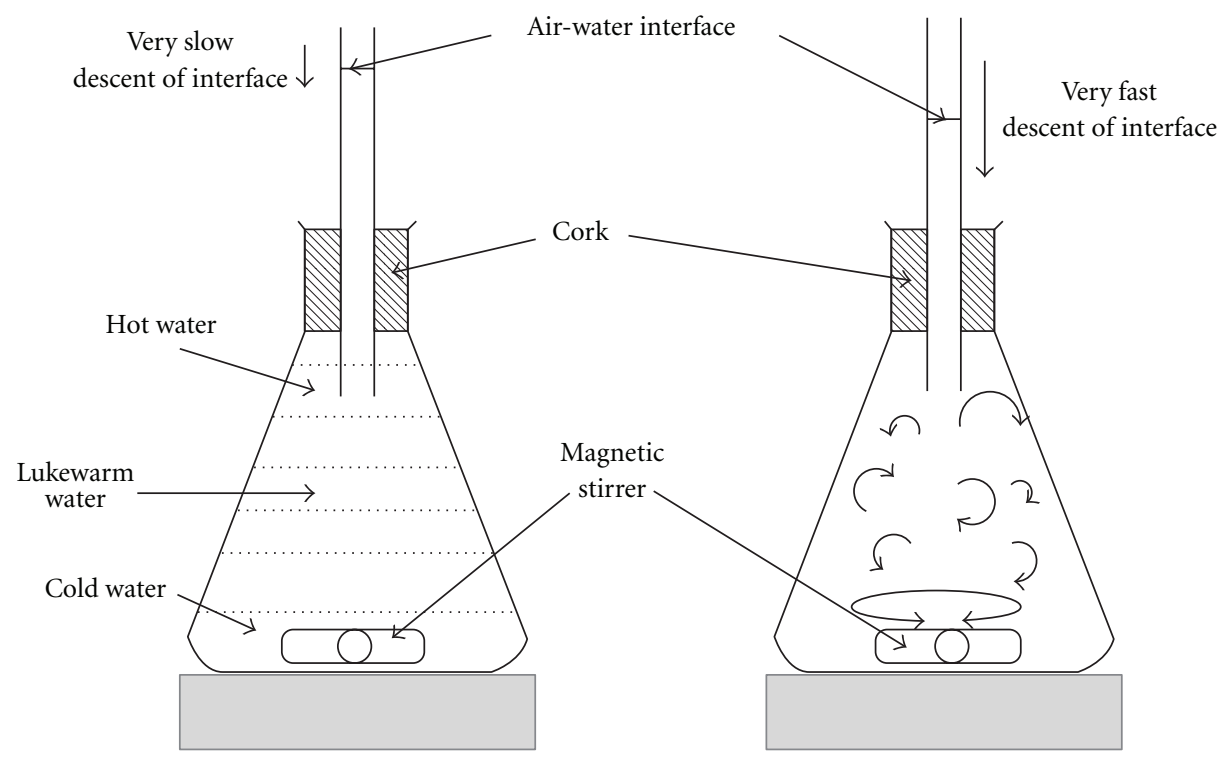

(a) Laminar evolution

(b) Turbulent evolution

FIGURE 2: Experimental setup of an experiment showed to the author by Peter Rhines during a visit to his GFD Laboratory in Seattle in June 2008, suggesting that compressibility effects increase with the degree of turbulent in stably stratified fluids. An Erlenmeyer flask is filled up with water at room temperature. A vertical stratification is then created by bringing the upper part of the water near boiling point, and a magnetic stirrer is dropped in the fluid. The top of the flask is then sealed up with a cork through which a thin glass tube is inserted in order to magnify the variations of the air-water interface. In laminar conditions (a), the fluid is near resting conditions, and the air-water interface moves downward very slowly as the result of molecular diffusion and/or radiative cooling. Activating the magnetic stirrer generates stratified turbulence (b) that results in the spectacular drop of the air-water interface, most likely as the result of the well-known contraction upon mixing effect that is due to the temperature dependence of the thermal expansion coefficient.

of the dynamics/thermodynamics coupling, and of the compressible work, comes from that in such a context the mechanical energy balance reduces to a balance between the compressible work and the total viscous dissipation, namely,

$$
\int_{V} P \frac{D v}{D t} \mathrm{~d} m=\int_{V} \varepsilon_{K} \mathrm{~d} m
$$

where $\mathrm{d} m=\rho \mathrm{d} V$ is the mass of an elementary fluid element. The central question here is to what extent is it possible to quantify the compressible work in (61), and whether the resulting estimates are sensitively affected by using the Boussinesq/Anelastic approximation as compared with using the fully compressible Navier-Stokes equations.

5.1. Antiturbulence Theorem and E-Theorems. The above issue was first addressed for a Boussinesq fluid with a linear equation of state governed by (41), as part of the derivation of the so-called antiturbulence theorem by Paparella and Young [17], whose particular aim was to derive an upper bound for the total viscous dissipation $\int_{V} \varepsilon_{K} \mathrm{~d} m$. Paparella and Young [17] derived their result for a two-dimensional rectangular ocean forced at its top by a Dirichlet-like surface temperature boundary condition, with insulating boundary conditions everywhere else. For the particular system of equations considered, the local balance equations for the kinetic energy and gravitational potential energy are then, respectively, given by

$$
\begin{gathered}
\rho_{0} \frac{D}{D t} \frac{\mathbf{v}^{2}}{2}+\nabla \cdot\left(P \mathbf{v}-\frac{\nu \nabla \mathbf{v}^{2}}{2}\right)=-\rho g_{0} w-\rho_{0} \varepsilon_{K}, \\
\frac{D\left(\rho g_{0} z\right)}{D t}=\rho g_{0} w+g_{0} z \frac{D \rho}{D t} .
\end{gathered}
$$

For a steady state, summing these two equations, and integrating over the whole domain yields

$$
\int_{V} \mathrm{~g}_{0} z \frac{D \rho}{D t} \mathrm{~d} V=\underbrace{\int_{V} g_{0} z \kappa \nabla^{2} \rho \mathrm{d} V}_{W_{\text {laminar }}}=\int_{V} \rho_{0} \varepsilon_{K} \mathrm{~d} V .
$$

The diffusive term $W_{\text {laminar }}$ has been previously encountered. As a result, such a balance can be written:

$$
W_{\text {laminar }}=\kappa g_{0} A\left[\langle\rho\rangle_{\text {bottom }}-\langle\rho\rangle_{\text {top }}\right]=\int_{V} \rho_{0} \varepsilon_{K} .
$$

Paparella and Young [17] showed that for the boundary conditions considered, the maximum principle imposes to the quantity $\langle\rho\rangle_{\text {bottom }}-\langle\rho\rangle_{\text {top }}$ to be bounded from above by a constant independent of the molecular diffusivity $\kappa$ and viscosity $\nu$. This result implies that $\int_{V} \varepsilon_{K} \mathrm{~d} m$ must converge toward zero in the limit of zero diffusivity by holding the Prandtl number $P_{r}=\nu / \kappa$ constant, which is the so-called antiturbulence theorem. For the present purposes, the result 
is of interest, because it provides an explicit expression of the work of expansion/contraction in terms of quantities that are all observable or measurable. Thus, Wang and Huang [18] used typical oceanic values to estimate that $B=O(15 \mathrm{GW})$ based on (64), which is about two orders of magnitude smaller than the power input due to the wind, generally estimated to be $O(1 \mathrm{TW})$. This result has been very influential, because it has generally been concluded that the observed amount of viscous dissipation in the oceans can only be accounted for by the mechanical stirring due to the wind and tides. It has also been regarded as implying that surface buoyancy fluxes can only drive a weak overturning circulation. The latter inference, however, requires that it is possible to link in some way the volume-integrated viscous dissipation, which only measures the amount of kinetic energy present at the dissipation scale, to the amount of kinetic energy of the large-scale overturning circulation. As far as we know, such a link is yet to be established rigorously.

5.2. Generalisation to a Nonlinear Equation of State. The equation of state of seawater is strongly nonlinear, however, so that it is unclear whether (64) remains valid for the more realistic oceanic case. The following provides a brief overview of various attempts at generalising (64) to a nonlinear equation of state typical of that for seawater.

5.2.1. Unsuitability of the Direct Approach. The most natural approach to estimating the volume-integrated work of expansion/contraction for a compressible ocean with a nonlinear equation of state is perhaps to regard the specific volume as a function of entropy and pressure (we discard salinity temporarily for simplicity), which allows one to expand the rate of expansion/contraction as follows:

$$
\begin{aligned}
\frac{D v}{D t} & =\frac{\partial v}{\partial \eta} \frac{D \eta}{D t}+\frac{\partial v}{\partial P} \frac{D P}{D t} \\
& =\frac{\alpha T}{\rho c_{p}} \frac{\left[\nabla \cdot\left(\kappa \rho c_{p} \nabla T\right)+\rho \varepsilon_{K}\right]}{\rho T}-\frac{1}{\rho^{2} c_{s}^{2}} \frac{D P}{D t},
\end{aligned}
$$

and therefore the volume-integrated work of expansion/contraction as follows:

$$
\begin{aligned}
B= & \underbrace{\int_{V} \frac{\alpha P}{\rho c_{p}} \nabla \cdot\left(\kappa \rho c_{p} \nabla T\right) \mathrm{d} V}_{B_{1}}+\underbrace{\int_{V} \frac{\alpha P}{\rho c_{p}} \rho \varepsilon_{K} \mathrm{~d} V}_{B_{2}} \\
& -\underbrace{\int_{V} \frac{P}{\rho c_{s}^{2}} \frac{D P}{D t} \mathrm{~d} V}_{B_{3}},
\end{aligned}
$$

where the expression was obtained by using the results that $\partial v / \partial \eta=\alpha T /\left(\rho c_{p}\right)=\Gamma$ is the so-called adiabatic lapse rate, and $\partial v / \partial P=\partial(1 / \rho) / \partial P=-\left(1 / \rho^{2}\right) \partial \rho / \partial P=-1 /\left(\rho^{2} c_{s}^{2}\right)$, where $c_{s}$ is the speed of sound. We also assumed the evolution equation for entropy to be given by

$$
\frac{D \eta}{D t}=\frac{\nabla \cdot\left(\rho \kappa c_{p} \nabla T\right)+\rho \varepsilon_{K}}{\rho T}
$$

which assumes that the only terms contributing to the diabatic heating are the molecular diffusion of heat and the viscous dissipation. Equation (66) regards $B$ as being made up of essentially three contributions, where $B_{1}$ is the net effect due to molecular diffusive heating/cooling, $B_{2}$ is the net effect due to viscous dissipation, and $B_{3}$ represents the net effect due to the adiabatic expansion/contraction. The two first terms $B_{1}$ and $B_{2}$ can be seen to involve the key dimensionless quantity $\Upsilon=\alpha P /\left(\rho c_{p}\right)$, which plays the role of a thermodynamic efficiency-like quantity. In the oceans, typical values are $\alpha=10^{-4} \mathrm{~K}^{-1}, P \approx 4000 \mathrm{dbar}=4.10^{8} \mathrm{~Pa}$ (near the ocean bottom), $\rho \approx 10^{3} \mathrm{~kg} \cdot \mathrm{m}^{-3}, c_{p} \approx 4.10^{3} \mathrm{~J} \cdot \mathrm{kg}^{-1} \cdot \mathrm{K}^{-1}$, leading to $\Upsilon \approx \times 10^{-2} \ll 1$. Since $B$ must balance the overall viscous dissipation $\int_{V} \rho \varepsilon_{K} \mathrm{~d} V$ in a steady-state, it follows immediately that the term $B_{2}$ must be negligible compared to the other two terms. Obviously, the term $B_{1}$ is the only one that can be compared with Paparella and Young [17] expression, since it is the only one depending on molecular diffusion. By integrating by parts, this term can be rewritten:

$$
\begin{aligned}
B_{1}= & \int_{\partial V} \Upsilon \kappa \rho c_{p} \nabla T \cdot \mathbf{n} \mathrm{d} S-\int_{V} \frac{\partial \Upsilon}{\partial T} \kappa \rho c_{p}\|\nabla T\|^{2} \mathrm{~d} V \\
& -\int_{V} \frac{\partial \Upsilon}{\partial P} \kappa \rho c_{p} \nabla T \cdot \nabla P \mathrm{~d} V .
\end{aligned}
$$

Note here that Paparella and Young [17] result can be recovered by assuming $\rho, c_{p}, \kappa$ to be constant, and the pressure to be approximated by the hydrostatic pressure $P \approx$ $-\rho_{0} g z$, as in that case, $\partial \Upsilon / \partial T=0$, while $\partial \Upsilon / \partial P=\alpha /\left(\rho c_{p}\right)$, and the boundary term vanishes owing to the assumption that $\int_{\partial V} \kappa \rho c_{p} \nabla T \cdot \mathbf{n} \mathrm{d} S=0$ required by the steady-state assumption. Ultimately, however, such a direct approach appears to be impractical, because it is unclear how to estimate the term $B_{3}$.

5.2.2. Approach Based on the Budget of Total Available Energy. A significant breakthrough in understanding how to get around the difficulty posed by the term $B_{3}$ above is due to McIntyre [19] and Nycander [9], which both showed that it could be circumvented by focusing on the budget of the total available energy $\mathbf{v}^{2} / 2+h^{\ddagger}$ discussed above. The following briefly summarises the key steps of their approach, which were derived for a Boussinesq ocean with a nonlinear equation of state. Here, we extend their discussion to the more general BA system discussed previously. To that end, let us first recall that in the BA system, the local evolution equation for the kinetic energy takes the form:

$$
\rho_{0} \frac{D}{D t} \frac{\mathbf{v}^{2}}{2}+\nabla \cdot\left[\delta P \mathbf{v}-\rho_{0} \mathbf{F}_{k e}\right]=\rho_{0} b \frac{D Z}{D t}-\rho_{0} \varepsilon_{K}
$$

In their derivation, both McIntyre [19] and Nycander [9] assumed the buoyancy $b=b(\mathrm{~S}, \Theta, Z)$ to be a given function of salinity $S$, conservative temperature $\Theta$, and geopotential 
height $Z$. Moreover, $\Theta$ and $S$ were assumed to satisfy evolution equations of the form:

$$
\begin{gathered}
\rho_{0} \frac{D \Theta}{D t}=-\nabla \cdot\left(\rho_{0} \mathbf{F}_{\Theta}\right)+\rho_{0} \dot{\Theta}_{\mathrm{irr}}, \\
\rho_{0} \frac{D S}{D t}=-\nabla \cdot\left(\rho_{0} \mathbf{F}_{S}\right),
\end{gathered}
$$

with $\mathbf{F}_{\Theta}$ and $\mathbf{F}_{S}$ being linear functions of the molecular diffusive fluxes of heat and salt, whose forms are traditionally constrained by the second law of thermodynamics, as discussed in de Groot and Mazur [20], for instance. The specific form of these fluxes is not important for what follows, and is therefore not further detailed. The term $\dot{\Theta}_{\text {irr }}$ represents the nonconservative production/destruction of the conservative temperature, which McDougall [21] argued is small enough to be regarded as negligible. As a result, this term was neglected by both McIntyre [19] and Nycander [9]. As discussed by Tailleux [22], however, this introduces some inconsistency in the discussion, as the resulting equations are no longer energetically consistent. This is a minor difficulty, however, which could easily be corrected by retaining $\dot{\Theta}_{\text {irr }}$. To proceed, McIntyre [19] and Nycander [9] introduced the following function:

$$
h^{\ddagger}(S, \Theta, Z)=-\int_{Z_{0}}^{Z} b\left(S, \Theta, Z^{\prime}\right) \mathrm{d} Z^{\prime},
$$

which was coined "dynamic enthalpy" by Young [8]. Differentiating with respect to time yields

$$
\frac{D h^{\ddagger}}{D t}=-b \frac{D Z}{D t}+C_{S} \frac{D S}{D t}+C_{\Theta} \frac{D \Theta}{D t}
$$

where

$$
\begin{aligned}
C_{S} & =-\int_{Z_{0}}^{Z} \frac{\partial b}{\partial S}\left(S, \Theta, Z^{\prime}\right) \mathrm{d} Z^{\prime}, \\
C_{\Theta} & =-\int_{Z_{0}}^{Z} \frac{\partial b}{\partial \Theta}\left(S, \Theta, Z^{\prime}\right) \mathrm{d} Z^{\prime} .
\end{aligned}
$$

As a result, summing (69) and (72) yields

$$
\begin{gathered}
\rho_{0} \frac{D}{D t}\left(\frac{\mathbf{v}^{2}}{2}+h^{\ddagger}\right)+\nabla \cdot\left(\delta P \mathbf{v}-\rho_{0} \mathbf{F}_{k e}\right) \\
\quad=\rho_{0}\left(C_{S} \frac{D S}{D t}+C_{\Theta} \frac{D \Theta}{D t}-\varepsilon_{K}\right) .
\end{gathered}
$$

The final step consists in integrating the latter equation over the whole ocean domain. In a steady-state, this leads to the following budget:

$$
\begin{aligned}
\int_{V} \rho_{0} \varepsilon_{K} \mathrm{~d} V= & B=-\int_{V}\left(C_{S} \nabla \cdot\left(\rho_{0} \mathbf{F}_{S}\right)+C_{\Theta} \nabla \cdot\left(\rho_{0} \mathbf{F}_{\Theta}\right)\right) \mathrm{d} V \\
= & -\underbrace{\int_{\partial V} \rho_{0}\left(C_{S} \mathbf{F}_{S}+C_{\Theta} \mathbf{F}_{\Theta}\right) \cdot \mathbf{n} \mathrm{d} S}_{=0} \\
& +\int_{V} \rho_{0}\left(\mathbf{F}_{S} \cdot \nabla C_{S}+\mathbf{F}_{\Theta} \cdot \nabla C_{\Theta}\right) \mathrm{d} V
\end{aligned}
$$

where the first term in the second line of (75) relates to the surface fluxes of $\mathbf{F}_{\Theta}$ and $\mathbf{F}_{S}$, which vanishes because by construction, $C_{\Theta}=C_{S}=0$ at the ocean surface where $Z=Z_{0}$. In McIntyre [19] and Nycander [9], the focus is on deriving upper bounds for $\int_{V} \rho_{0} \varepsilon_{K} \mathrm{~d} V$, but clearly the method also provides a way to discuss the magnitude of $B$, since the two quantities must be equal in a steady state. The important point to be noted here is that (75) provides an expression for $B$ that only involves the molecular diffusive fluxes, with no term proportional to $D P / D t$, similarly as in Paparella and Young [17] study.

5.2.3. Approach Based on the Budget of Potential Enthalpy. In this section, we aim to further clarify the above issues, by showing how the above results can be extended to a fully compressible ocean by considering the budget of potential enthalpy. To simplify the discussion, the effects of salinity are discarded. The potential enthalpy, introduced in the oceanographic context by McDougall [21], is defined as the enthalpy that a fluid parcel would have if brought adiabatically to the reference pressure $P_{0}$, usually taken as the standard atmospheric pressure. As a result, the potential enthalpy, denoted by $h_{\theta}$ thereafter, can be regarded as being implicitly defined by $h_{\theta}\left(\eta, P ; P_{0}\right)=h\left(\eta, P_{0}\right)$, with $h$ being the specific enthalpy. From the definition of its total differential $\mathrm{d} h=$ $T \mathrm{~d} \eta+v \mathrm{~d} P$, it follows that

$$
\frac{D h_{\theta}}{D t}=\theta \frac{D \eta}{D t}=\frac{\theta}{T}\left[\nabla \cdot\left(\kappa \rho c_{p} \nabla T\right)+\rho \varepsilon_{K}\right],
$$

where $\theta$ is the potential temperature, that is, the temperature that a parcel would have if brought adiabatically to the ocean surface, and hence defined as the implicit solution of $\eta(T, P)=\eta\left(\theta, \mathrm{P}_{0}\right)$ or as the solution of $\eta(T, S, P)=$ $\eta\left(\theta, S, P_{0}\right)$ if salinity was to be retained. Now, if we take the overall budget in a steady state, we have the result:

$$
\begin{gathered}
\underbrace{\int_{\partial V} \kappa \rho c_{p} \nabla T \cdot \mathbf{n} \mathrm{d} S}_{=0}-\underbrace{\int_{V} \kappa \rho c_{p} \nabla T \cdot \nabla\left(\frac{\theta}{T}\right) \mathrm{d} V}_{\dot{h}_{\mathrm{irr}}} \\
+\int_{V} \frac{\theta}{T} \rho \varepsilon_{K} \mathrm{~d} V=0,
\end{gathered}
$$

which was obtained by noting that $\theta=T$ at the reference pressure $P=P_{0}$. Here, it is useful to introduce the quantity:

$$
s=\frac{\int_{V}((T-\theta) / T) \rho \varepsilon_{K} \mathrm{~d} V}{\int_{V} \rho \varepsilon_{K} \mathrm{~d} V} .
$$

Physically, $\theta$ never differs more than of a few degrees Celsius from the in-situ temperature, which implies $s \ll 1$. As a result, it follows that (77) implies $\int_{V} \rho \varepsilon_{K} \mathrm{~d} V=\dot{h}_{\text {irr }} /(1-s) \approx$ $\dot{h}_{\text {irr }}$, which in turn implies

$$
B \approx \dot{h}_{\mathrm{irr}}=\int_{V} \kappa \rho c_{p} \nabla T \cdot \nabla\left(\frac{\theta}{T}\right) \mathrm{d} V .
$$

To make progress, note that from the definition of potential temperature $\eta(T, P)=\eta\left(\theta, P_{0}\right)$, we may regard $\theta$ as 
a function of temperature and pressure. In particular, using the result that $\mathrm{d} \eta=\left(c_{p} / T\right) \mathrm{d} T-(\alpha / \rho) \mathrm{d} P$, it follows that

$$
\frac{c_{p}}{T} \mathrm{~d} T-\frac{\alpha}{\rho} \mathrm{d} P=\frac{c_{p}\left(\theta, P_{0}\right)}{\theta} \mathrm{d} \theta,
$$

which implies

$$
\frac{\partial \theta}{\partial T}=\frac{c_{p} \theta}{c_{p}^{r} T}, \quad \frac{\partial \theta}{\partial P}=-\frac{\alpha \theta}{\rho c_{p}^{r}},
$$

where for simplicity, we defined $c_{p}\left(\theta, P_{0}\right)=c_{p}^{r}$. Now, using (81) leads to

$$
\begin{aligned}
\nabla\left(\frac{\theta}{T}\right) & =\frac{T \nabla \theta-\theta \nabla T}{T^{2}} \\
& =\frac{1}{T}\left[\frac{\partial \theta}{\partial T} \nabla T+\frac{\partial \theta}{\partial P} \nabla P\right]-\frac{\theta \nabla T}{T^{2}} \\
& =\frac{1}{T}\left[\frac{c_{p} \theta}{c_{p}^{r} T} \nabla T-\frac{\alpha \theta}{\rho c_{p}^{r}} \nabla P\right]-\frac{\theta \nabla T}{T^{2}} \\
& =\left(\frac{c_{p}-c_{p}^{r}}{c_{p}^{r}}\right) \frac{\theta \nabla T}{T^{2}}-\frac{\alpha \theta}{\rho c_{p}^{r} T} \nabla P,
\end{aligned}
$$

which in turn allows one to express $\dot{h}_{\text {irr }}$ as follows:

$$
\begin{aligned}
\dot{h}_{\text {irr }}= & \underbrace{\int_{V} \theta\left(\frac{c_{p}-c_{p}^{r}}{c_{p}^{r}}\right) \frac{\kappa \rho c_{p}\|\nabla T\|^{2}}{T^{2}} \mathrm{~d} V}_{H_{1}} \\
& -\underbrace{\int_{V} \frac{\alpha c_{p} \theta}{c_{p}^{r} T} \kappa \nabla P \cdot \nabla T \mathrm{~d} V .}_{H_{2}}
\end{aligned}
$$

Equation (83) is a key result, as it currently represents the most general extension of Paparella and Young [17] result that is valid for a compressible ocean with a nonlinear equation of state. Although salinity was not considered, the result could be easily extended to include it as well. As in Paparella and Young [17], McIntyre [19], and Nycander [9], the expression obtained only involves terms related to molecular diffusive processes. It is composed of mainly two terms $H_{1}$ and $H_{2}$. The first term involves the quantity $R=$ $\theta\left(c_{p}-c_{p}^{r}\right) / c_{p}^{r}$, which can be shown to be in general negative for most conditions encountered in the oceans. It is zero at the surface, and increase with pressure. By invoking the intermediate value theorem, it follows that there exists a value $R^{*}$ so that $H_{1}$ can be written as follows:

$$
H_{1}=R^{*} \int_{V} \frac{\kappa \rho c_{p}\|\nabla T\|^{2}}{T^{2}} \mathrm{~d} V<0 .
$$

Interestingly, under this form, $H_{1}$ appears as being proportional to the irreversible entropy production by molecular diffusive heat fluxes. Since $H_{1}$ is negative, $H_{2}$ must also be negative in order for $B$ to positive in order to balance the overall viscous dissipation. Some insight into $\mathrm{H}_{2}$ can be obtained by rewriting it as follows:

$$
\begin{aligned}
H_{2} & =\int_{V}\left(\frac{c_{p} \theta}{c_{p}^{r} T}\right) \alpha \kappa \nabla P \cdot \nabla T \mathrm{~d} V \\
& =\int_{V} \frac{c_{p} \theta}{c_{p}^{r} T} \alpha \kappa \nabla_{h} P \cdot \nabla_{h} T \mathrm{~d} V+\int_{V} \frac{c_{p} \theta}{c_{p}^{r} T} \alpha \kappa \frac{\partial P}{\partial z} \frac{\partial T}{\partial z} \mathrm{~d} V .
\end{aligned}
$$

Interestingly, it is the last term in (85) that allows one to make the connection with Paparella and Young [17] result, as seen by the following manipulation:

$$
\begin{aligned}
\int_{V} \frac{c_{p} \theta}{c_{p}^{r} T} \alpha \kappa \frac{\partial P}{\partial z} \frac{\partial T}{\partial z} \mathrm{~d} V & \approx-\int_{V} \alpha \rho g_{0} \kappa \frac{\partial T}{\partial z} \mathrm{~d} V \\
& =\int_{V} \kappa g \frac{\partial \rho}{\partial z} \mathrm{~d} V=-W_{\text {laminar }}
\end{aligned}
$$

by approximating $c_{p} \theta /\left(c_{p}^{r} T\right) \approx 1$ and using the hydrostatic approximation $\partial P / \partial z \approx-\rho g_{0}$. It is more difficult, however, to quantity the correlation between the horizontal pressure and temperature gradients $\nabla_{h} P \cdot \nabla_{h} T$; presently, even the sign of the later, let alone its magnitude, is challenging to estimate, because the correlation between the two gradients is likely to occur at the molecular diffusive scales. Somehow, understanding how to quantify this term is equivalent to understanding the characteristics of the turbulent pressure and temperature fluctuations at such small diffusive scales. More research is needed to clarify this issue.

5.3. General Remarks on Deriving Expressions for B. Reflecting in hindsight on the different ideas that have been developed over the past decade to generalise Paparella and Young [17] result to a fully compressible ocean with a nonlinear equation of state, it appears that the most general approach to estimating the viscous dissipation and hence the overall work of expansion/contraction is ultimately rooted in the steady-state budget for some function $F(\eta)$ of the specific entropy. Indeed, if we consider the evolution equation for such a function, it is given by

$$
\rho \frac{D F(\eta)}{D t}=\rho F^{\prime}(\eta) \frac{D \eta}{D t}=F^{\prime}(\eta) \frac{\nabla \cdot\left(\kappa \rho c_{p} \nabla T\right)+\rho \varepsilon_{K}}{T} .
$$

Integrating this equation over the ocean volume and assuming a steady state thus yield

$$
\int_{V} \frac{F^{\prime}(\eta) \nabla \cdot\left(\kappa \rho c_{p} \nabla T\right)}{T} \mathrm{~d} V+\int_{V} \frac{F^{\prime}(\eta) \rho \varepsilon_{K}}{T} \mathrm{~d} V=0 .
$$

Now, if $F(\eta)$ is chosen as an increasing function of $\eta$ so that $F^{\prime}(\eta)>0$, then the theorem of intermediate values implies 
that there must exist some value $\eta^{*}$ and $T^{*}$ allowing to rewrite the above formula as follows:

$$
\begin{aligned}
\int_{V} \rho \varepsilon_{K} \mathrm{~d} V=-\frac{T^{*}}{F^{\prime}\left(\eta^{*}\right)} \int_{V} \frac{F^{\prime}(\eta) \nabla \cdot\left(\kappa \rho c_{p} \nabla T\right)}{T} \mathrm{~d} V \\
=-\frac{T^{*}}{F^{\prime}\left(\eta^{*}\right)}\left\{\int_{\partial V} \frac{F^{\prime}(\eta) \kappa \rho c_{p} \nabla T \cdot \mathbf{n}}{T} \mathrm{~d} S\right. \\
\left.-\int_{V} \kappa \rho c_{p} \nabla T \cdot \nabla\left(\frac{F^{\prime}(\eta)}{T}\right) \mathrm{d} V\right\} .
\end{aligned}
$$

Clearly, this method appears to systematically provide an expression for the viscous dissipation as the sum of one term involving the surface heat fluxes and one term linked to the irreversible molecular diffusive fluxes. Clearly, the method based on potential enthalpy derived above is based on the particular choice $F(\eta)=h_{\theta}\left(\eta, P ; P_{0}\right)$ and has the particularity that the term involving the surface fluxes identically vanish. Two different particular choices corresponding to $F(\eta)=\eta$ and to $F(\eta)=h\left(\eta, P_{r}(\eta)\right)$ for some reference pressure $P_{r}(\eta)$ related to Lorenz [15] reference profile were considered by Tailleux [2] as part of a discussion on the nature of the work of expansion/contraction. The current challenge consists in identifying the most sensible choices of $F(\eta)$, and to determine what kind of information can be extracted from making such choices. To that end, however, the main difficulties is in understanding how to quantify the kind of correlation between the pressure and temperature gradients that enter such expressions, which requires understanding the nature of such gradients at the molecular diffusive scales that are likely to control the overall value of such a correlation.

\section{Summary and Conclusions}

For over a century, the coupling between the dynamics and thermodynamics has been overwhelmingly regarded as being of little or no relevance to the understanding of stratified turbulence in weakly compressible fluids such as water or seawater. As a result, the study of such fluids has been nearly systematically been carried out in the context of dynamical approximations, such as the Boussinesq approximation or anelastic approximation, that decouples the dynamics and thermodynamics at leading order. It is not necessarily fully realized, however, that such a decoupling is achieved only in absence of diabatic effects and for a linear equation of state. Indeed, coupling is unavoidably reintroduced whenever any one of the latter effects is retained. If so, a key issue is what can we say about the nature of such a coupling in that case? How does it compare with that of the fully compressible Navier-Stokes equations?

In order to address these questions, the present paper first showed that the particular anelastic approximation derived by Pauluis [6], which admits the Boussinesq approximation as a particular case, can be endowed with a fully consistent energetics and thermodynamics, even for a binary fluid with an arbitrary nonlinear equation of state. As showed in more details by Tailleux [10], it is not only possible to show that the energetics of such a system possesses a term that can be directly interpreted as the approximation to the work of expansion/contraction, it is also possible to construct explicitly the full range of thermodynamic potentials for such a fluid. This is an important result, because it permits for the first time to clarify the role played by internal energy in the Boussinesq/Anelastic system, which has been previously a large source of confusion, owing to the "incompressible" label attached to the Boussinesq approximation, or to the notion that such approximations filter out sound waves, which is often interpreted as the lack of a coupling with internal energy. In particular, the clarification allows one to confirm the previous results by Tailleux [1], who argued that the dissipation of available potential energy, previously interpreted by Winters et al. [14] as an irreversible conversion of APE into background gravitational potential energy, should in fact be regarded as an irreversible conversion of APE into internal energy, in the same way that viscous dissipation is irreversible dissipation of mechanical energy into internal energy. Finally, we also determined how to extend the derivation of so-called $\varepsilon$-theorems for horizontal convection to the case of a fully compressible fluid with an arbitrary nonlinear equation of state.

An important feature of the energetics of the BA system is that there is a priori no physical mechanism to prevent the conversion term between internal energy and mechanical energy to become potentially very large. In other words, even though the BA system is constructed under the premises that compressibility effects are small, it is now clear that the BA compressible effects will in general increase with the strength of the stratification and turbulence. In principle, the magnitude of the compressibility effects can be diagnosed $a$ posteriori by estimating the divergent velocity field $\mathbf{v}_{d}$ from solving the equation $\nabla \cdot \mathbf{v}_{d}=-\rho^{-1} D \rho / D t$. For instance, in the case of the Boussinesq model with a diffusive model for the density, this would consist in solving $\nabla \cdot \mathbf{v}_{d}=-\kappa \nabla^{2} \rho / \rho$, which makes it clear that $\mathbf{v}_{d}$ can become large if the local values of diffusion $\kappa \nabla^{2} \rho$ become large, which is expected to be the case as the degree of turbulence and strength of the stratification increase. Further work is needed to determine whether it may become possible for $\mathbf{v}_{d}$ to become sufficiently large as to become locally important in the advection of momentum and tracers. If so, this would also potentially suggest the importance of sound waves. As is well known, acoustic waves can induce mean flows, as in the case of acoustic streaming. Whether such effects may be identified in stratified turbulence opens up an exciting new terrain of investigation.

\section{References}

[1] R. Tailleux, "On the energetics of stratified turbulent mixing, irreversible thermodynamics, Boussinesq models and the ocean heat engine controversy," Journal of Fluid Mechanics, vol. 638, pp. 339-382, 2009.

[2] R. Tailleux, "Entropy versus APE production: on the buoyancy power input in the oceans energy cycle," Geophysical Research Letters, vol. 37, no. 22, Article ID L22603, 2010. 
[3] G. O. Hughes and R. W. Griffiths, "Horizontal convection," Annual Review of Fluid Mechanics, vol. 40, pp. 185-208, 2008.

[4] J. Boussinesq, Théorie Analytique de la Chaleur, vol. 2, Gauthiers-Villars, Paris, France, 1903.

[5] Y. Ogura and N. A. Phillips, "Scale analysis and shallow convection in the atmopshere," Journal of the Atmospheric Sciences, vol. 19, pp. 173-179, 1962.

[6] O. Pauluis, "Thermodynamic consistency of the anelastic approximation for a moist atmosphere," Journal of the Atmospheric Sciences, vol. 65, no. 8, pp. 2719-2729, 2008.

[7] A. P. Ingersoll, "Boussinesq and anelastic approximations revisited: Potential energy release during thermobaric instability," Journal of Physical Oceanography, vol. 35, no. 8, pp. 1359 1369, 2005.

[8] W. R. Young, "Dynamic enthalpy, conservative temperature, and the seawater Boussinesq approximation," Journal of Physical Oceanography, vol. 40, no. 2, pp. 394-400, 2010.

[9] J. Nycander, "Horizontal convection with a non-linear equation of state: generalization of a theorem of Paparella and Young," Tellus, vol. 62, no. 2, pp. 134-137, 2010.

[10] R. Tailleux, "Thermodynamics/dynamics coupling and thermodynamic consistency of Boussinesq and anelastic binary fluids with an arbitrary nonlinear equation of state," http:// arxiv.org/abs/1011.5071.

[11] H. B. Callen, Thermodynamics and an Introduction to Thermostatistics, Wiley, 1985.

[12] R. A. Alberty, "Use of legendre transforms in chemical thermodynamics," Pure and Applied Chemistry, vol. 73, no. 8, pp. 1349-1380, 2001.

[13] R. Feistel, "A new extended Gibbs thermodynamic potential of seawater," Progress in Oceanography, vol. 58, no. 1, pp. 43-114, 2003.

[14] K. B. Winters, P. N. Lombard, J. J. Riley, and E. A. D’Asaro, "Available potential energy and mixing in density-stratified fluids," Journal of Fluid Mechanics, vol. 289, pp. 115-128, 1995.

[15] E. N. Lorenz, "Available potential energy and the maintenance of the general circulation," Tellus, vol. 7, pp. 157-167, 1955.

[16] O. Pauluis, "Sources and sinks of available potential energy in a moist atmosphere," Journal of the Atmospheric Sciences, vol. 64, no. 7, pp. 2627-2641, 2007.

[17] F. Paparella and W. R. Young, "Horizontal convection is nonturbulent," Journal of Fluid Mechanics, vol. 466, pp. 205-214, 2002.

[18] W. Wang and R. X. Huang, "An experimental study on thermal circulation driven by horizontal differential heating," Journal of Fluid Mechanics, vol. 540, pp. 49-73, 2005.

[19] M. E. McIntyre, "On spontaneous imbalance and ocean turbulence: generalisations of the Paparella-Young epsilon theorem," in Proceedings of the International IUTAM/Newton Institution Workshop, D. G. Dritschel, Ed., Turbulence of the Atmosphere and Oceans, Springer, 2010.

[20] S. R. de Groot and P. Mazur, Non-Equilibrium Thermodynamics, North Holland, 1962.

[21] T. J. McDougall, "Potential enthalpy: a conservative oceanic variable for evaluating heat content and heat fluxes," Journal of Physical Oceanography, vol. 33, no. 5, pp. 945-963, 2003.

[22] R. Tailleux, "Identifying and quantifying nonconservative energy production/destruction terms in hydrostatic Boussinesq primitive equation models," Ocean Modelling, vol. 34, no. 3-4, pp. 125-136, 2010. 

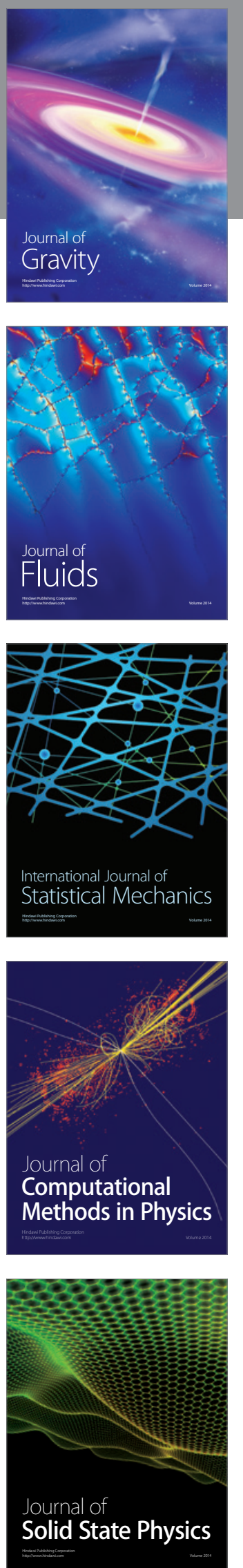
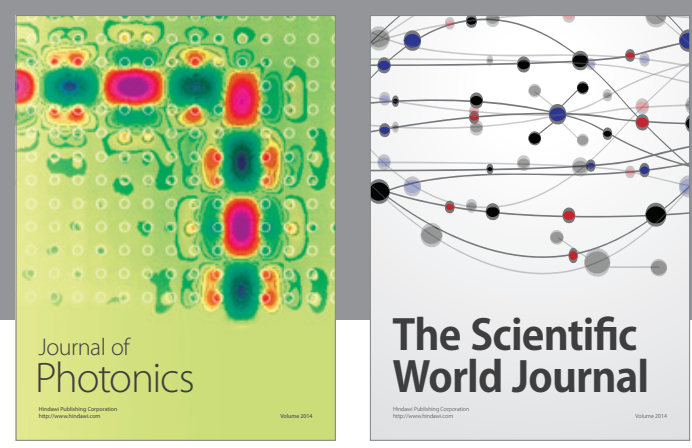

The Scientific World Journal

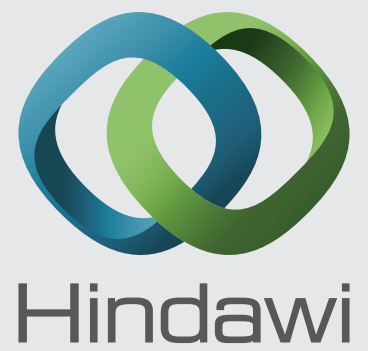

Submit your manuscripts at http://www.hindawi.com
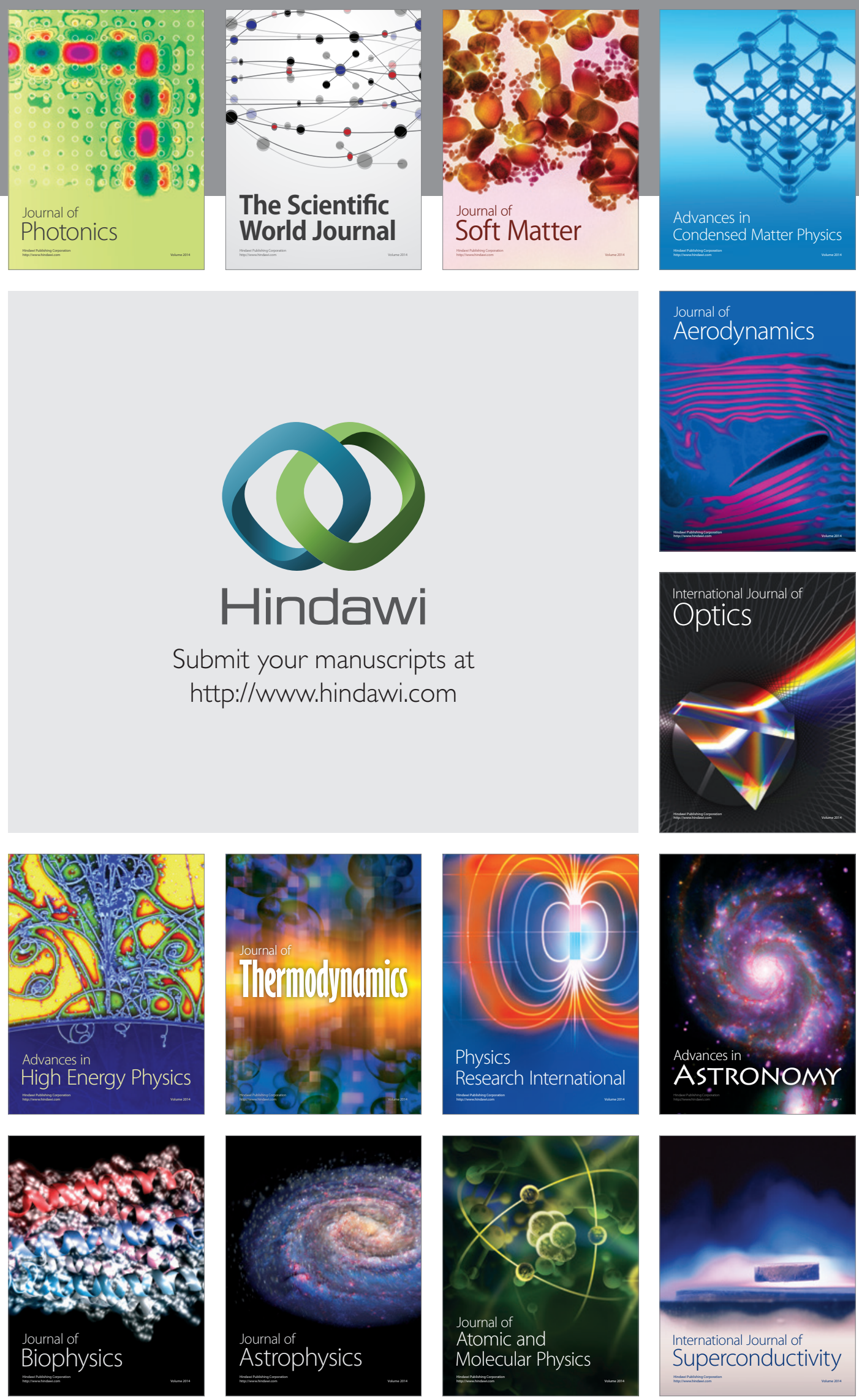
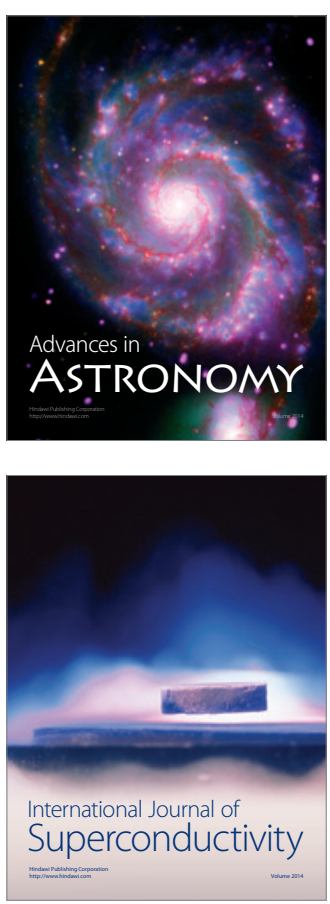